\title{
Políticas públicas y Estado en Brasil: consecuencias económicas, sociales y ambientales en el desarrollo del municipio de Sorriso en Mato Grosso (Brasil)
}

\author{
Lázaro Camilo Recompensa Joseph \\ Universidad Federal de Mato Grosso, Cuiabá, Brasil. \\ Email: camilojoseph@hotmail.com
}

Benedito Dias Pereira

Universidad Federal de Mato Grosso, Cuiabá, Brasil.

Email: bdp@terra.com.br

\author{
Vanisa Raquel Scheuer Graff \\ Universidad Federal de Mato Grosso, Cuiabá, Brasil. \\ Email: vanisaraquel@hotmail.com \\ Rosana Guimaraes \\ Universidad Federal de Mato Grosso, Cuiabá, Brasil. \\ Email: rosaeconomia@gmail.com
}

\begin{abstract}
Resumen: Este artículo busca describir y analizar de manera general los diferentes mecanismos y elementos de la política pública que facilitan el movimiento y/o la expansión de los agricultores de la soja, en el Estado de Mato Grosso y específicamente en la municipalidad de Sorriso, y las principales consecuencias económicas, sociales y ambientales. El trabajo está estructurado en cuatro partes. La primera caracteriza y describe los principales mecanismos con que la política pública favorece la expansión y/o ocupación de la soja en el Estado de Mato Grosso. La segunda muestra las principales consecuencias sociales y medioambientales que acompañan al modelo de producción. La tercera plantea la necesidad de "políticas públicas de promoción" y modelos organizacionales como arreglos, innovaciones y sistemas productivos, que consideren las condiciones económicas locales. Finalmente se exponen las conclusiones.
\end{abstract}

Palabras clave: Políticas públicas y estrategia de desarrollo, territorios y espacios, sociedad civil y sistemas productivos e innovativos locales 


\title{
Public policies and State in Brazil: economic, social and environmental impacts in the development of the municipality of Sorriso in Mato Grosso (Brazil)
}

\begin{abstract}
This paper aims to describe and analyze in general the different mechanisms and elements of public policy that "facilitated" the movement and / or expansion of soybean farmers in the State of Mato Grosso, specifically in the municipality of Sorriso; and what were the main economic, social and environmental impacts. The work is structured in four parts. The first characterizes and describes the main mechanisms of public policy that favored the expansion and / or occupation of soy in Mato Grosso State, the second highlights the main environmental and social consequences attached to this model of production, the third places the need for "public policy advocacy" and organizational models as arrangements, and innovative and productive systems capable of generating local economic, social and environmental conditions. And finally the conclusions are presented.

Key words: Public policy and development strategy, territories, space, civil society and local productive and innovative systems

\section{Políticas públicas e Estado no Brasil: desenvolvimento económico, social e ambiental do município de Sorriso, em Mato Grosso (Brasil)}

Resumo: O presente artigo pretende descrever e analisar os diferentes mecanismos de elementos de política pública que facilitam a circulação e / ou expansão dos fazendeiros de soja no estado de Mato Grosso e, especificamente no município de Sorriso, e as principais consequências econômicas, sociais e ambientais. O trabalho está organizado em quatro partes. O primeiro caracteriza e descreve os principais mecanismos que a política pública favorece a expansão e / ou ocupação de soja em Mato Grosso. O segundo mostra as principais consequências sociais e ambientais que acompanham o modelo de produção. O terceiro levanta a necessidade de “defesa de políticas públicas” e os modelos organizacionais, tais como arranjos, inovações e sistemas de produção que consideram as condições econômicas locais. Finalmente, apresenta as conclusões.

Palavras-chave: desenvolvimento de políticas e estratégias públicas, territórios e espaço, sociedade civil, sistemas produtivos locais e inovadoras

$* * *$

\section{Introducción}

Según Bickel (2004) la rápida expansión de la producción de soja se debió fundamentalmente a la infraestructura construida con los subsidios otorgados por el gobierno brasilero. El régimen militar instalado a partir de 1964 promovió la ocupación y modernización agrícola de las regiones Centro Oeste y Norte. Este proceso de expansión (en el Estado de Mato Grosso) significo la instrumentación de un modelo de producción favorable a una elite rural (tanto en sus inicios como en la actualidad), modelo que negligencia los aspectos sociales y ambientales del desarrollo. 
El presente trabajo tiene como objetivo describir y analizar de forma general los diferentes mecanismos y elementos de política pública que "facilitaron" el movimiento y/o expansión de los productores de soja en el Estado de Mato Grosso, específicamente en el municipio de Sorriso y cuáles fueron sus principales consecuencias económicas sociales y ambientales.

El trabajo está estructurado en cuatro partes. En la primera se caracteriza y se describe los principales mecanismos de política pública que favorecieron a la expansión y/o ocupación de la soja en el Estado de Mato Grosso, la segunda destaca las principales consecuencias sociales y ambientales acopladas a ese modelo de producción, la tercera coloca la necesidad de busca de "políticas públicas de promoción” y modelos organizacionales como los arreglos y sistemas productivos e innovativos locales $^{1}$ capaces de generar condiciones de desarrollo económico, social y ambiental. Y por último se colocan diferentes consideraciones finales.

\section{Metodología de la investigación}

Para describir y analizar de forma general los diferentes mecanismos y elementos de políticas públicas que "facilitaron" el movimiento y/o expansión de los productores de soja en el Estado de Mato Grosso en específico en el municipio de Sorriso, se utilizaron fuentes informativas primarias y secundarias, o sea, se realizo un levantamiento de las principales bibliografías existentes, trabajos científicos, tesis, informativos, publicaciones técnicas, datos de la RAIS e informaciones obtenidas vía internet. Al mismo tiempo se realizó una investigación de campo en el municipio de Sorriso, a través de la cual efectuaron se entrevistas y se aplicó un cuestionario previamente elaborado (con base en investigaciones realizadas anteriormente) y relacionadas con el tema de análisis del trabajo. Estas entrevistas nos ofrecieron los elementos necesarios para una mejor comprensión del problema tratado en el municipio.

La estratificación según el tamaño fue realizada a partir del número de trabajadores empleados obtenida de la RAIS del Ministerio del Trabajo y Empleo del Estado de Mato Grosso para el municipio seleccionado. El tamaño de la empresa es definido de acuerdo con los siguientes parámetros:

\begin{tabular}{|c|c|}
\hline Estrato & Tamaño según el número de trabajadores \\
\hline Micro & Hasta 19 trabajadores \\
\hline Pequeños & 20 a 99 trabajadores \\
\hline Medio & 100 a 499 trabajadores \\
\hline Grande & Más de 500 trabajadores \\
\hline
\end{tabular}

El procedimiento utilizado para la estratificación de la muestra fue el siguiente: se calculó la población (N) y el tamaño mínimo de la muestra 
para los diferentes productores de soja del municipio de Sorriso, utilizando un intervalo de confianza de $80 \%$, o sea, debido a que el universo de la investigación presentaba un valor (N: =456) muy alto. Siendo así, se aplicaron 21 cuestionarios entre los diferentes empresarios de la soja en el municipio.

$$
\begin{aligned}
& \text { Datos } \\
& N=456 \\
& E_{0}=0,22 \\
& \text { Calculando } \mathrm{n} \\
& n=\frac{N * n_{0}}{N+n_{0}-1}=\frac{456 * 21}{456+(21-1)}=21 \\
& N=\text { poblacion } \\
& n=\frac{1}{\left(E_{0}\right)^{2}}=\frac{1}{(0,22)^{2}}=21 \\
& n=\text { tamano minimo de la muestra } \\
& E_{0}=\text { Error muestral aceptable }
\end{aligned}
$$

\section{Principales mecanismos de política pública que favorecieron a la expansión/ocupación de la soja en el Estado de Mato Grosso}

En este ítem describiremos de forma general los diferentes mecanismos y elementos de la política pública que facilitaran el movimiento/expansión de los productores de soja en el Estado y en el municipio de Sorriso. Según Bickel (2004), la rápida expansión de la soja se dio por la infraestructura construida con los recursos monetarios otorgados por el gobierno brasileño. El régimen militar instalado a partir de 1964 promovió la ocupación y la modernización agrícola de las regiones del Centro Oeste, Norte y Nordeste. En Minas Gerais, por ejemplo, el Programa de Crédito Integrado (PCI, 1971) transfirió recursos a los grandes y medios propietarios para su respectiva modernización agrícola. Paralelamente al PCI fue instaurado el primer "Programa de Asentamiento Dirigido", en Alto Paranaiba, Minas Gerais (PADAP), donde se entregaron 60 mil hectáreas para la explotación agrícola intensiva ${ }^{2}$. El Gobierno de Minas Gerais gastó aproximadamente US\$ 200 millones en la construcción de carreteras, centros rurales, almacenes y habitaciones, líneas de transferencias de crédito, así como incentivos para la intensificación de la producción como el uso de maquinas e insumos agrícolas.

El PADAP fue el modelo para los dos nuevos programas de desarrollo regional, el Programa para el Desarrollo del Cerrado (POLOCENTRO) y el Programa de Cooperación Nipo Brasileño para el Desarrollo del Cerrado (PROCEDER). El POLOCENTRO (1975-1982) tenía como objeti- 
vo incorporar 3,7 millones de hectáreas de Cerrados (1,8 millones para la agricultura, 1,2 millones para la pecuaria y 700 mil para reforestar) a la actividad agrícola. Además se destinaron US\$ 577 millones a los sectores de almacenamiento, energía, investigación, asistencia técnica, crédito rural subsidiado, utilización intensiva de correctivos de suelo, mecanización, transporte, electrificación rural etc. El POLOCENTRO benefició en su gran mayoría a los productores con propiedades mayores de 200 hectáreas, este programa en vez de reservar $60 \%$ del área para la producción de alimentos, dedicó la mayor parte del área a la producción de pastos y de soja para la exportación (Bickel, 2004).

El PRODECER promovió a partir de 1978 el asentamiento de agricultores experientes del sudeste y sur del país. Apoyado en un acuerdo entre los gobiernos brasileños y japonés, se origino el interés de asegurar la fuente de las importaciones japonesas de soja y de estimular la agricultura moderna en los Cerrados. La Compañía de Promoción Agrícola (CAMPO), fundada con el 51\% de capital nacional y 49\% de capital japonés, fue la encargada de esta ejecución. Así con una inversión total de US\$ 570 millones el PROCEDER fue instaurado en tres fases en los Estados de Minas Gerais, Mato Grosso, Mato Grosso del Sur, Goias, Bahia, Tocantins y Maranhao.

Se ejecutaron 21 proyectos con 758 asentados, incorporando 353.748 hectáreas de cerrados al proceso productivo ${ }^{3}$. Las políticas de crédito subsidiado implantadas entre 1970 y 1980 beneficiaron significativamente a los agricultores modernos y políticamente más influyentes. Pero la inflación y la elevación de los intereses durante las turbulencias económicas de los años 80 generaron un mayor endeudamiento de parte de los beneficiarios del PROCEDER II y III, con la recesión y el necesario ajuste macro económico de los años 80 la disponibilidad de crédito fueron gradualmente reducidas.

Debemos destacar que en 1975 se creó la Empresa Brasileña de Investigación Agropecuaria (EMBRAPA) con la función de viabilizar el cultivo de la soja en todo el Brasil y la EMBRAPA cerrados como centro de investigación regional con foco en el bioma cerrado y cuyo objetivo era generar y difundir tecnologías para la ocupación regional de la región. Hasta ese momento, la producción de soja se restringía a los Estados de la región tradicional productora de soja en el país y a los cultivares utilizados en la época provenientes de los Estados Unidos. Los trabajos de investigación fueron completados con las empresas de investigación agropecuaria estatales, en las cuales prevalecía como estándar de referencia de la época, el uso de tecnología de la llamada "revolución verde", o sea, la aplicación de tecnologías intensivas en el uso de capital apoyadas en la mecanización y en el uso intensivo de insumos externos como fertilizantes químicos y agrotóxicos (Ibid).

Para divulgar estas tecnologías y auxiliar a los productores se creó la Empresa de Asistencia Técnica y Extensión Rural (EMATER). 
Dada la creciente escasez de recursos públicos, la EMBRAPA recorre a la realización de cooperación y asociación con las instituciones de investigación privadas de las empresas e industria con intereses específicos como por ejemplo con la multinacional Monsanto y fundaciones norteamericanas.

En los años 80 la estrategia de desarrollo de los cerrados fue fundamentada y articulada a través de una política de soporte de precios, o sea, para compensar la eliminación de los subsidios al crédito rural exigida por el Banco Mundial y el FMI, el Gobierno aumento considerablemente los precios mínimos, que anteriormente se establecían abajo del nivel del mercado, pasando así a adquirir volúmenes expresivos de productos oriundos del PROCEDER, como soja, arroz y maíz.

Esto beneficio a los productores de las áreas más remotas de los cerrados, para quienes los precios del mercado eran menores por el elevado costo del transporte. Por otro lado el gobierno sufrió pérdidas substanciales dado los diferenciales de precios, los altos costos del transporte, almacenaje y el deterioro de los productos. Finalmente con la implementación en los años 90 de las políticas neoliberales de ajuste estructural, la política de compras y stocks gubernamentales fueron eliminadas.

Estos programas fueron y están siendo acompañados por inmensas inversiones públicas en la construcción de puertos, carreteras, hidro-vías y líneas de ferrocarril con el objetivo de disminuir los costos de la exportación y la producción de granos. A pesar del amplio consenso existente en relación a mejorar la infra estructura, diversos institutos de investigación ambiental alertan para los grandes impactos socio económicos y ambientales negativos generados por estas políticas públicas: por ejemplo el asfaltado de la carretera BR-163 de Cuiabá a Santarém, podría provocar una gran desforestación, la cual afectaría severamente las tierras indígenas, parques nacionales, el equilibrio acuático, la biodiversidad, y el clima regional y global (Nepstad, 2000) .

Hasta hace pocos años atrás, los bancos públicos federales y estatales (Banco de Desarrollo, Banco da Amazonia, Banco del Nordeste, Fondos Constitucionales, etc.) ofrecían programas de crédito a intereses subsidiados (8,75\% a 10,75\% al año) para financiar las actividades de la agricultura patronal. Esto significa que el comportamiento del crédito rural en Brasil ha tenido un papel preponderante tal como se puede ver en la tabla 1.

Es decir, la participación del BNDES registra una disminución en los últimos tres años/zafras analizados, siendo que esta baja es compensada con el aumento de la participación del Banco de Brasil en el crédito rural, que en los últimos cinco años tiene un aumento de 85,57\%. 


\section{Tabla 1}

Gastos destinados al Crédito Rural (Inversión y costo) de 2002 a 2007 (Año/Zafras ${ }^{4}$ )

\begin{tabular}{cccccc}
\hline Bancos / Zafra & Jul 02/ & Jul 03/ & Jul 04/ & Jul 05/ & Jul 06/ \\
& Jun 03 & Jun 04 & Jun 05 & Jun 06 & Jun 07 \\
BNDES & 3,5361 & 5,521 & 5,751 & 3.334 & 2.911 \\
& & & & & \\
BANCO DO & 29,974 & 39,710 & 48,376 & 51.038 & 55.624 \\
BRASIL & & & & & \\
\hline Participación del & $11,8 \%$ & $13,9 \%$ & $11,9 \%$ & $6,5 \%$ & $5,2 \%$ \\
BNDES & & & & & \\
\hline
\end{tabular}

Fuente: Banco Nacional de Desenvolvimento (BNDES)

Los programas agrícolas del Plan de Zafra del Gobierno Federal, ejecutados con los recursos del BNDES, constituyen uno de los principales canales de financiamiento del Banco al sector. El valor desembolsado para estos programas agrícolas en el año 2006 y en el periodo de 2007 fueron respectivamente $\mathrm{R} \$ 4,5$ billones y $\mathrm{R}$ \$ 2,6 millones. A continuación destacamos estos programas:

El Programa de Desarrollo Cooperativo para la Agregación de Valor a la Producción Agropecuaria (PRODECOOP), destinado a las cooperativas de producción agropecuaria y a cooperados para la integración de cuotas partes vinculadas al proyecto a ser financiado, tiene como objetivo el incremento de la competitividad del complejo agroindustrial de las cooperativas, mediante la modernización de los sistemas productivos y de comercialización. A través de este programa fueron liberados en 2006 aproximadamente R\$ 166 millones para un total de 113 operaciones, siendo el valor medio de las operaciones de R\$ 1,5 millones. En el primer semestre de 2007 fueron liberados R\$ 129 millones para un total de 59 operaciones, con valor medio de R\$ 2,2 millones;

El Programa de Modernización de la Agricultura y Conservación de Recursos Naturales (MODERAGRO): destinados a los productores rurales (personas físicas y jurídicas) y sus cooperativas con el objetivo de incentivar la corrección del suelo, la recuperación de áreas de pastos degradadas, la sistematización de micro presas con la intención de aumentar la producción de granos y la implantación de proyectos de adecuación ambiental de propiedades rurales. Mediante ese programa fue liberado en $2006 \mathrm{R} \$ 2,2$ billones para un total de 12.044 operaciones con un valor medio de R\$ 183 mil. En el primer semestre de 2007 se otorgaron R\$ 527 
millones para un total de 6.072 operaciones lo que represento un valor medio de R\$ 88.791 por operaciones de crédito.

El Programa de Modernización de la Flota de Tractores Agrícolas e Implementos Asociados y Cosechadoras, (MODERFROTA): destinado a los productores rurales, personas físicas o jurídicas y sus cooperativas. a través de este programa se otorgaron en 2006, R\$1,3 billones para un total de 14.412 operaciones y en el primer semestre de 2007 R\$ 845 millones para un total de 8.382 operaciones de crédito.

\section{El Programa de Incentivo a la Irrigación y el almacenaje} (MODERINFRA) destinado a los productores rurales, personas físicas o jurídicas y sus cooperativas cuyo objetivo era apoyar el desarrollo de la agricultura sostenible disminuyendo los riesgos en la producción y aumentar la oferta de alimentos para el mercado interno y externo y ampliar la capacidad de almacenamiento en las propiedades rurales. Para este programa se desembolso en 2006 R \$ 200 millones para un total de 3.935 operaciones y en el primer semestre de 2007 se liberaron R\$ 76 millones en 425 operaciones de crédito.

Debemos destacar que pasado diez años de un gobierno que estimuló e implementó la mayor desnacionalización jamás vista en el Brasil, el camino quedó pavimentado para la actuación y dominio de cuatro grandes empresas transnacionales, la Cargill, Bunge, ADM y la Dreyfus (a través de la empresa Coimbra) las cuales dominan aproximadamente el 95\% de las exportaciones de la soja brasileña.

Estas tradings que en la actualidad pertenecen (su gran mayoría) a las empresas multinacionales que producen agrotóxicos e insumos agrícolas ocuparon el vacío dejado por el Estado años atrás, ocupando y desempeñando diferentes funciones como la de prestar dinero a los agricultores con intereses gigantescos. La forma de financiamiento utilizada por estas multinacionales, (además de ofertar la logística y los almacenes) para dominar a los productores, pueden ser clasificadas en dos: la primera es la realizada a través de las operaciones de soja verde (esto es la venta anticipada del producto) y la segunda es el cambio de fertilizantes por granos como es realizado por la Bunge y la Cargill, ambas fabricantes de abonos y fertilizantes.

En el caso especifico del Estado de Mato Grosso, el 63\% de la soja producida in natura es destinada para la exportación y como determinado por la Ley Kandir ${ }^{5}$ esta commodity es exonerada del pago de cualquier tipo de impuesto cuando es destinada a la exportación: siendo así las tradings no pagan absolutamente nada y su contribución directa para el desarrollo sostenible del territorio y del municipio de Sorriso es prácticamente nula. La tabla 2 nos muestra la participación de estas empresas en el comercio de Brasil. 
Tabla 2

Ranking de las empresas exportadoras e importadoras del agronegocio brasileño en 2007

\begin{tabular}{|c|c|c|c|c|c|c|}
\hline & \multicolumn{3}{|c|}{ EXPORTADORAS } & \multicolumn{3}{|c|}{ IMPORTADORAS } \\
\hline & \begin{tabular}{|l|} 
EMPRESA \\
\end{tabular} & $\begin{array}{l}\text { EXPORTACIONES } \\
\text { En millones de dólares } \\
\text { FOB. }\end{array}$ & $\begin{array}{l}\text { PARTICIPACION } \\
\text { (en \% do total) }\end{array}$ & EMPRESA & $\begin{array}{l}\text { IMPORTACIONES } \\
\text { En millones de } \\
\text { dólares FOB. }\end{array}$ & $\begin{array}{l}\text { PARTICIPACION } \\
\text { (en \% del total) }\end{array}$ \\
\hline 10 & $\begin{array}{l}\text { Bunge } \\
\text { Alimentos }\end{array}$ & 3055 & 1,90 & $\begin{array}{l}\text { Bunge } \\
\text { Alimentos }\end{array}$ & 470 & 0,39 \\
\hline 20 & SADIA & 1776 & 1,11 & $\begin{array}{l}\text { ADM do } \\
\text { Brasil - } \\
\text { Archer } \\
\text { Daniels } \\
\text { Midland }\end{array}$ & 334 & 0,28 \\
\hline 30 & $\begin{array}{l}\text { Cargill } \\
\text { Agricola }\end{array}$ & 1759 & 1,10 & AMBEV & 205 & 0,17 \\
\hline 40 & Perdigão & 1393 & 0,87 & $\begin{array}{l}\text { Cargill Agri- } \\
\text { cola }\end{array}$ & 189 & 0,16 \\
\hline 50 & $\begin{array}{l}\text { Louis } \\
\text { Dreyfus } \\
\text { (Brasil) }\end{array}$ & 1280 & 0,80 & $\begin{array}{l}\text { M. DIAS } \\
\text { BRANCO }\end{array}$ & 181 & 0,15 \\
\hline 60 & $\begin{array}{l}\text { ADM do } \\
\text { Brasil - } \\
\text { Archer } \\
\text { Daniels } \\
\text { Midland }\end{array}$ & 1224 & 0,76 & SADIA & 134 & 0,11 \\
\hline
\end{tabular}

Fuente: Anuario de comercio exterior de 2008

\section{Consecuencias sociales y ambientales de la concentración de los productores de soja en el Estado de Mato Grosso y en el municipio de Sorriso}

En el ítem anterior describimos los diferentes elementos de política pública que facilitaron la expansión de los productores de soja en el Estado de Mato Grosso, a continuación analizaremos las consecuencias sociales y ambientales resultados de la aplicación de la misma. En primer lugar explicaremos de forma general la evolución de ese proceso de movimiento/expansión (en el Estado de Mato Grosso) que significó la aplicación de un modelo de producción favorable a una elite rural desde el inicio de estos programas hasta los días de hoy. Esta elite acumula numerosas ventajas recibidas de los gobiernos federales estatales y municipales, como por ejemplo la facilidad en la adquisición de tierras estatales: siendo esta la responsable por la creación de los inmensos latifundios en el Estado y de la concentración de tierras y de la riqueza existente hoy día en el municipio.

O sea, con el golpe militar de 1964 se instaló en Brasil una dictadura a servicio del capital monopolista, subordinando el mismo a la agricultura. En ese contexto no existía espacio para realizar una reforma agraria que 
disminuyera las desigualdades sociales, en la mayoría de los casos lo que se notó fue la creación de asentamientos rurales para fijar la mano de obra barata cerca de las haciendas. Se facilitaron incentivos fiscales y préstamos para ocupar las llamadas "fronteras agrícolas" que beneficiaron fundamentalmente las grandes propiedades rurales.

Según Reydon y Cornelio (2006) este es el núcleo del problema agrario en Brasil el cual tiene sus orígenes en el modelo de ocupación y de desarrollo del país que en su génesis no paso por la ruptura de la "sagrada alianza" entre la burguesía y los propietarios de la tierra. O sea, el proceso de modernización de la economía brasileña no rompió con las fuerzas más retrogradas manteniendo el poder político concedido a los propietarios de tierra.

Las evidencias de que el conjunto de las regulaciones en relación con la tierra en el Brasil fueron establecidas para beneficiar a los propietarios de tierras pueden ser comprobadas por la permanencia de la "pose o posesión” como figura jurídica fundamental de la apropiación de la tierra, siendo esta una de las conquista de los propietarios de la tierra, ya que la misma permite la incorporación de las nuevas áreas con costos bastantes reducidos (Ibid).

Además de la alternativa de la pose y con el pretexto de que era necesario poblar regiones "sin habitantes", el Estado invirtió grandes cantidades de recursos públicos para crear la infraestructura necesaria para esto como: carreteras, hidroeléctricas, facilidades de crédito, subsidios estatales y permisos para especular con la tierra. Este tipo de ocupación / desarrollo en el caso del Estado de Mato Grosso permitió que se ignorara a las comunidades tradicionales, indígenas y a los poseeros que ya habitaban en aquellos territorios, proceso que favoreció y posibilito el desforestamiento de gran parte del Amazonas.

Según Mançano (2004) con la introducción del concepto de agronegocio y la construcción de su imagen tratase renovar a la agricultura capitalista e intentase mostrar a la misma como "moderna". O sea con este "nuevo" concepto, los propietarios de grandes extensiones rurales intentan vincular su figura e imagen a la de modernas empresas agrícolas, cuyos lucros y resultados impulsan el buen desempeño de la balanza comercial brasileña.

El agro-negocio como conjunto de actividades que abarcan la producción agropecuaria y forestal, el beneficio para sus productos y subproductos, la agro-industrialización, las empresas productoras de insumos (semillas, agrotóxicos, fertilizantes, maquinas, tractores etc.), el comercio de esas mercancías, el sistema bancario relacionado con el crédito rural y el seguro agrícola, están siendo reducido por la propaganda dominante a la esfera de aquellos productos y subproductos directamente relacionados con la exportación y negociación en la Bolsa Mercantil y de Futuros (BM\&F) como por ejemplo el maíz (grano y aceite) la soja (grano, 
aceite y torta), los porcinos, las aves, los bovinos (carne, cuero, pieles), los productos forestales (madera, papel, pasta de celulosa). O sea, el agronegocio en la ideología dominante es sinónimo de agro-negocio burgués (Carvalho, 2004).

En este sentido la propaganda capitalista destaca subliminarmente que son los grandes productores los responsables por la producción de esas mercancías y que esas mercancías abarcan la totalidad del agronegocio, negando así la existencia de "agronegocios democráticos y populares” (Ibid).

Debemos destacar entre las principales consecuencias ambientales la deforestación que en los últimos 30 anos (de 1978 a 2008) alcanzó un ritmo anual entre 20.000 y $201.799 \mathrm{~km}^{2}$, lo que representa en media $6.300 \mathrm{~km}^{2}$ por año, lo que equivale a la superficie de 555.555 campos de futbol por año en el Estado de Mato Grosso (ver tabla 3)

Tabla 3

Superficie total desforestada en $\mathbf{K m}^{2}$ en el Estado de Mato Grosso.

\begin{tabular}{cccc}
\hline ANO & $\begin{array}{c}\text { SUPERFICIE } \\
\left.\text { DESFORESTADA (KM }{ }^{2}\right)\end{array}$ & $\begin{array}{c}\text { INDICE DE } \\
\text { DESFORESTAMENTO } \\
\text { ANUAL (KM }{ }^{2} / \text { ANO) }\end{array}$ & $\begin{array}{c}\text { NUMERO DE } \\
\text { VICTIMAS } \\
\text { FATALES EN EL } \\
\text { CAMPO }\end{array}$ \\
1978 & & & - \\
1988 & 20000 & 5140 & 1 \\
1989 & 71500 & 5960 & 3 \\
1990 & 79600 & 4020 & 4 \\
1991 & 83600 & 2840 & 0 \\
1992 & 84220 & 4674 & 2 \\
1994 & 86500 & 6220 & 3 \\
1995 & 91174 & 6220 & 4 \\
1996 & 103614 & 10391 & 2 \\
1997 & 112150 & 6543 & 2 \\
1998 & 119141 & 5271 & 1 \\
1999 & 125023 & 6466 & 0 \\
2000 & 136968 & 6963 & 4 \\
2001 & 146431 & 6369 & 2 \\
2002 & 153592 & 7703 & 9 \\
2003 & 163495 & 7892 & 2 \\
2004 & 174306 & 10405 & 5 \\
2005 & 184655 & 11814 & 2 \\
2006 & 193185 & 7145 & 1 \\
2007 & 195773 & 4333 & 2 \\
2008 & 198354 & 2678 & 2 \\
\hline & 201799 & 3258 & 2 \\
\hline
\end{tabular}

Fuente: PRODES, INPE 
Debemos destacar que existen diferentes tipos de deforestación: por tala rasa ${ }^{6}$ y por degradación forestal. La primera es aquella que resulta en la remoción completa de la cobertura forestal en corto intervalo de tiempo, es también conocida como "corta y quema" en la cual toda la vegetación es derrumbada y quemada ${ }^{7}$ en un pequeño intervalo de tiempo. En este proceso la cobertura forestal es totalmente removida y substituida por otras coberturas y usos (agrícolas, pastos, urbano, hidroeléctricas, etc). El proceso normalmente comienza antes o durante el período de lluvia que precede el corte de la floresta, es localmente denominado de "taladraje". Es el corte con hacha de los arboles menores y principalmente de las "ramas trepaderas" para posteriormente facilitar el corte de los arboles de mayor tamaño que se realizara en la próxima fase. Durante la época de lluvia esas plantas se degradan y con eso se evita accidentes en la fase de corte.

Las árboles de mayor porte son talados con moto sierras en el inicio del período seco, así permanece la biomasa en el suelo, que es quemada básicamente entre julio y septiembre. Posteriormente se forma el pasto por la siembra de gramíneas africanas que son resistentes al fuego y se desarrollan muy bien en el Amazonas. Ese fue el proceso que comúnmente se utilizó en el Estado de Mato Grosso durante las décadas del 80, 90 y comienzos del siglo XXI.

La deforestación por degradación forestal se caracteriza por la extracción de arboles de mayor valor económico antes de realizar los procedimientos de limpieza a través de las quemadas. Primeramente se retiran las maderas nobles, luego es plantado el pasto y en los años siguientes entran (en la floresta) el ganado y la pecuaria.

Las figuras 1, A, B y C reflejan las diferentes escalas de la degradación forestal originada por la extracción ilegal de madera. El caso "A" representa la degradación de intensidad moderada, esto es el área en regeneración después de la explotación maderera, la “B”, la degradación de alta intensidad, o sea, exploración maderera activa, grande proporción del suelo expuesta y la " $C$ ", la degradación de leve intensidad, la cual evidencia la abertura de carreteras y caminos de acceso.

La tabla 4 nos muestra la distribución de las áreas desforestadas en el Estado de Mato Grosso. Esos valores indican que existe una cantidad considerable de áreas en proceso de degradación que como ilustrado en la figura pueden convertirse en de corte raso si no se interrumpe este proceso.

Tabla 3

\section{Deforestación por corte raso y degradación forestal en 2007 y 2008.}

\begin{tabular}{lcc}
\hline \multicolumn{1}{c}{ Tabla en Mato Grosso: } & \\
\multicolumn{1}{c}{ Tipos de Desflorestamento } & $\mathbf{2 0 0 7}$ & $\mathbf{2 0 0 8}$ \\
Áreas degradadas $\left(\mathrm{km}^{2}\right)$ & 8.744 & 12.534 \\
Áreas degradadas $(\mathrm{km} 2)$ en 2007 convertidas a corte raso en & & 920 \\
2008 & & \\
\hline
\end{tabular}

Fuente: Instituto Nacional de Pesquisas Espaciais - INPE 
Es necesario destacar que en el 2004 el Instituto de Pesquisa Ambiental de La Amazonía (IPAM) publicó un libro titulado "Desmatamento na Amazonia: indo alem da emergencia crónica" en el cual clasifica la deforestación en apropiada e inapropiada.

La deforestación apropiada sería aquel que reúne los siguientes criterios: a) es realizado de forma legal, cumpliendo los preceptos de las leyes ambientales (por ejemplo el Código Forestal), b) es realizado en suelos productivos aptos para la agricultura, c) Ocurre en áreas con infra estructura adecuada y con bajo riesgo de abandono precoz de la actividad, d) aporta beneficios socioeconómicos y ambientales a las poblaciones tradicionales del Amazonas (indios y pequeños agricultores).

La deforestación inapropiada (que debe ser foco de los programas de reducción) es aquella que: a) tiene como objetivo tomar la posesión de la tierra para la especulación; b) ocurre en tierras inadecuadas para la agricultura o la ganadería debido al relieve accidentado, suelos impropios, localizados a grandes distancias de los mercados y ausencia de carreteras y caminos; c) es poco productiva, d) es ilegal (alcanza las reservas y las áreas de protección permanentes); e) ocurre en áreas de elevado valor para la conservación y/o utilización sostenible de la biodiversidad (áreas aun no protegidas por unidades de conservación); f) es realizada en áreas donde la mejor opción económica de uso de la tierra es forestal, sea para la producción maderera, no maderera, o ambas.

\section{Figura 1 \\ A, B y C representa los estándares de degradación forestal}

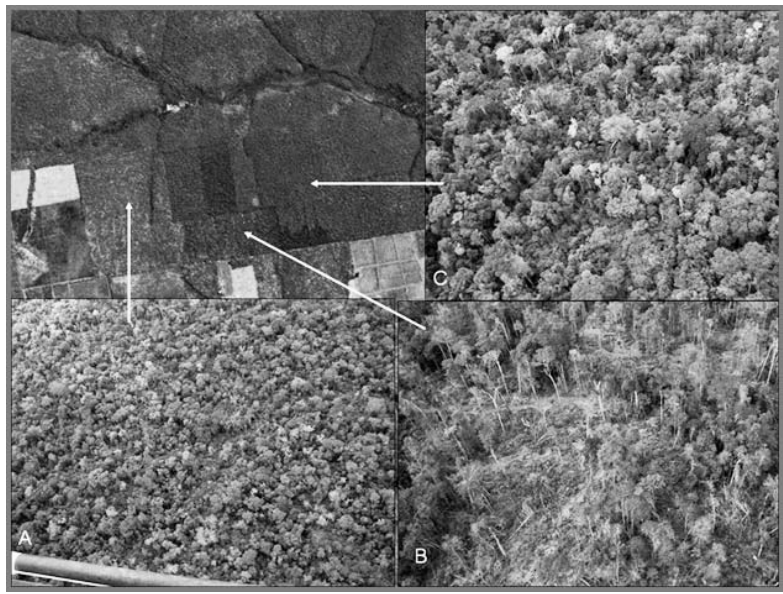

A partir de esta clasificación podemos separar la deforestación derivada de acciones de "pillaje" como aquella que afecta aceleradamente las regiones localizadas en las orillas de la carretera BR-163 en el Estado de 
Mato Grosso, donde se concentran varios municipios "campeones de la deforestación” de aquella deforestación permitida (dentro del código forestal) para cada propietario. El municipio de Sorriso es uno de los mas desforestado (aproximadamente más de la mitad de su superficie). La figura 2 nos muestra la deforestación acumulada en el Estado de Mato Grosso hasta 2004 y 2005.

\section{Figura 2 \\ Desflorestamiento en Mato Grosso (acumulado hasta Agosto 2004-2005)}

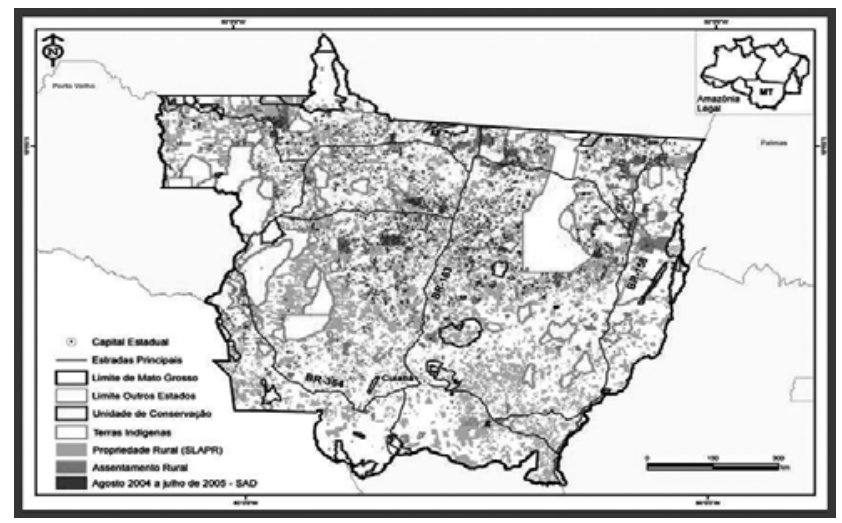

Fuente: Méndez Del Villar (2005)

Potencialmente tres agentes son los principales responsables por la desforestación del Estado y del municipio de Sorriso. O sea, el movimiento de expansión-ocupación de la frontera agrícola (dicho de otra forma el proceso de ocupación/desarrollo del territorio) sigue la siguiente lógica: primeramente se talan los árboles por parte de los madereros, posteriormente las áreas desforestadas son ocupadas con pastos para la cría de rebaño vacuno y, por último se cultiva con arroz (por un periodo de 2 a 3 años) cerrándose el ciclo con la siembra de soja.

En Brasil diferentes autores han intentado explicar el fenómeno de la desforestación, o sea, procurase saber quién es el principal responsable de este proceso: el maderero, el ganadero o el agricultor (plantador de soja). La mayoría de los estudios responsabiliza a los dos primeros como los principales "villanos" porque en este proceso de abertura y ocupación de nuevas áreas, parece que existe una articulación entre madereros y ganaderos, porque después de la explotación y retirada de la madera de interés comercial, los ganaderos encuentran mayor facilidad para penetrar y ocupar áreas en la floresta utilizando las carreteras y caminos abierto por los madereros.

Pero resulta que en el Estado de Mato Grosso debido a la frecuente ausencia del Estado o por la conveniencia del mismo y debido a la enorme 
extensión de tierras “desocupadas”, el pillaje (o la ocupación ilegal de tierras) le sigue posteriormente al maderero.

Debemos destacar que Douglas Morton (2006) especialista de la Universidad de Maryland, publicó un estudio en la revista de la Academia Nacional de Ciencias de EEUU, en el cual deja al descubierto a los agricultores (de soja) como uno de los principales agentes desforestadores en el Estado de Mato Grosso. En su trabajo destaca en primer lugar, que la soja no solamente ocupa áreas previamente desforestadas para pastos y posteriormente abandonadas por los ganaderos, o sea, los datos muestran ese detalle pero no es lo suficiente para explicar toda la dinámica de ese proceso.

En segundo lugar, destaca que las variaciones en los precios del grano solo se reflejan en los índices de desforestación, dos o tres años después de ocurrida, porque la transformación de la floresta en plantaciones agrícolas (de soja en este caso) lleva tiempo. El trabajo demostró que más del $90 \%$ de las aberturas para la agricultura fueron plantadas con soja en el primer año después de la desforestación (ver Folha de Sao Paulo, 05/09/2006).

O sea, cruzando imágenes del satélite y con levantamientos en el campo, Morton estimó en $5.400 \mathrm{~km}^{2}$ el total de la floresta convertida directamente para granos en el Estado de Mato Grosso entre 2001 y 2004. En el ano de 2003, cuando el precio de la soja en el mercado internacional alcanzó el pick, la conversión directa para la plantación represento aproximadamente un cuarto de todo lo que se desforesto en el Estado de Mato Grosso campeón en la época de la devastación del Amazonas (ver figura 3).

Haciendo una breve comparación esa superficie desforestada y sustituida con soja, equivale a casi la extensión del Distrito Federal, sin contar el efecto conocido del grano de soja de "empujar" la frontera agrícola indirectamente, estimulando a la pecuaria a ocupar nuevas áreas. En este período Mato Grosso desforestó 38 mil km², el equivalente a 3,5 veces el tamaño de Jamaica, lo que representó el $40 \%$ de todo lo que perdió de la floresta Amazónica (Ibid).

Figura 3

El efecto de la cultura de la soja en la desforestación del Estado de Mato Grosso 2001 - 2004

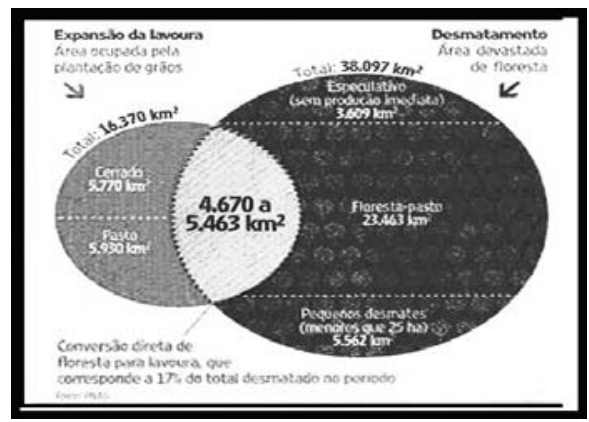

Fuente: PNAS 
Es decir, a través del trabajo de Morton (2006) se puede diferenciar la desforestación realizada para pasto y para plantaciones agrícolas, verificándose que las áreas dedicadas a la siembra de soja eran dos veces superior a las abiertas y dedicadas con la pecuaria ${ }^{8}$, lo que demuestra que existe una fuerte correlación entre el precio de la soja con el índice anual de deforestación (Ibid).

El estudio destaca que en ese período disminuyó en $12 \%$ la transformación de la floresta para pasto y un aumento de la floresta convertida en plantación en 10\%. Además en ese mismo periodo 2001-2004 Morton acompaño las emisiones de carbono después de la deforestación y llegó a la conclusión de que el avance del agro-negocio capitalizado y mecanizado sobre la floresta está agravando el efecto estufa en la región. En el caso de la transformación de la floresta para producir granos, las emisiones se suceden todas de una vez, ya que la agricultura mecanizada no admite "suciedad o desechos” y el secuestro de carbono por rebote es cero. Se trata según el autor de una reducción completa de la biomasa en un período de cuatro años (Ibid).

Recientemente Melo (2009) analizó cómo las diferentes actividades productivas del agro-negocio en Mato Grosso interfieren directamente sobre la desforestación, identificando que actividades fueron las responsables por la expansión de la frontera agrícola en los municipios del Estado de Mato Grosso en el período de 2001 a 2007.

Utilizando fuentes de información obtenidas en el Instituto Brasilero de Geografía y Estadísticas (IBGE), la Secretaria de Planificación de Mato Grosso (SEPLAN-MT), el Proyecto de Monitorización de la Floresta Amazónica por Satélite del Instituto Nacional de Pesquisa Espacial de Brasil (PRODES-INPE) entre otros y con el uso de las herramientas econométricas como el modelo de los coeficiente de correlación de Pearson, el método de análisis factorial y el método de cointegracion de Johansen, verificó que la producción de soja, maíz y vacunos son las actividades que en la actualidad más estimulan la desforestación en el Estado de Mato Grosso. La figura 4 sintetiza esos análisis.

En la meso región noreste del Estado de Mato Grosso (donde se encuentra localizado el municipio de Sorriso) la cual concentra la mayor área de floresta del Estado, la soja, el maíz y la pecuaria representan las actividades productivas que más presión directa ejercen sobre la desforestación en la región.

En la meso región norte, donde existe una de las mayores reservas indígenas del Brasil (el área que concentra la mayor parte de las floresta da región y del cerrado) los resultados allí obtenidos no indican la acción de ninguna actividad productiva sobre la desforestación. Debemos destacar que en esta meso región la desforestación existente es más del tipo de degradación forestal derivadas de la actividad maderera ilegal. 
En el suroeste del Estado, una región abierta (hace ya mucho tiempo) no se registra una gran incidencia de la desforestación en el período analizado. En la región centro-.sur del Estado de Mato Grosso (la cual no posee más floresta nativa) existe una fuerte acción de la caña de azúcar sobre el proceso de desforestación. Y por último en la región sureste los resultados indican que las actividades productivas como la pecuaria y la soja son las responsables por la desforestación en la región.

Figura 4

Distribución de la responsabilidad de la desforestación en las principales meso regiones en el Estado de Mato Grosso.

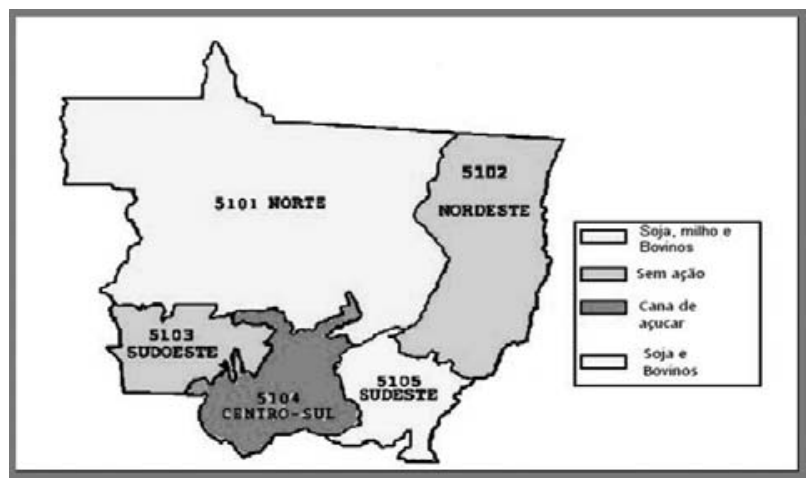

Fuente: Melo (2009)

Siendo así, es posible que no exista una fuerza principal que impulse o explique por sí sola la desforestación dentro del Estado, porque las causas son varias y se derivan de una combinación sofisticada de diversos factores. Esto es extremamente importante para poder entender que la dinámica de la desforestación del Estado está directamente asociada a tres elementos fundamentales: a) las políticas públicas que permitieron la conformación y evolución de los grandes latifundios agrícolas en la región; b) a las dinámicas productivas utilizadas cuya origen esta acoplada al modelo de ocupación/desarrollo (in)sostenible (económico, social y ambientalmente) implantado en Brasil en la década del 60/ 70; c) la impunidad, o sea, el no respeto al código forestal vigente desde 1965.

En el caso de particular de la región investigada, el municipio de Sorriso, dentro de las principales consecuencias ambientales observadas debemos destacar las siguientes:

La deforestación del mismo, derivada de la acción directa de la producción de soja. Hasta el 2007 se desforesto 3945 km², el equivalente al $42 \%$ del área total do municipio, sobrando de la floresta $1279,8 \mathrm{~km}^{2}$, lo que representa el 14\% del área total del municipio (ver tabla 4). 


\section{Tabla 4 \\ Área total y desforestación acumulada en el municipio de Sorriso hasta el año 2007}

\begin{tabular}{lcc}
\hline \multicolumn{1}{c}{ Desforestación } & \multicolumn{2}{c}{ Municipio Sorriso } \\
& $\mathbf{K m}^{\mathbf{2}}$ & $\mathbf{( \% )}$ \\
Área en $\mathrm{km}^{2}$ & 9350,0 & 100 \\
Desforestación & 3954,0 & $\mathbf{4 2}$ \\
Floresta* & 1279,8 & 14 \\
No floresta" $^{*}$ & 4115,5 & 44 \\
\hline
\end{tabular}

*Representa el acumulado hasta el 2007.

Fuente: PRODES-INPE

En el curso del arroyo central ${ }^{9}$ presencia de residuos sólidos lanzados (y no recogidos) indebidamente en las pendientes y en los propios cursos del agua, lo que representa serios riesgos a la salud colectiva, contaminación física, química y biológica del suelo y de las aguas superficiales y subterráneas, aumentando la probabilidad de inundaciones en razón de los disturbios que provocan en el funcionamiento hidráulico de las estructuras de drenaje cuando estos residuos son movidos y/o transportados por las aguas de lluvia; Desaparición de la vegetación del área que forma la naciente del arroyo y la cobertura vegetal debajo de lo que exige la Legislación Ambiental; degradación de la áreas de preservación permanentes; ocupación irregular de las orillas del arroyo; la urbanización y la implantación de la infra estructura removió varias especies autóctonas de su hábitat natural; la presencia de la erosión a lo largo del arroyo central, (donde la ciudad nació) este fenómeno está asociado a la ocupación de la ciudad; o sea, la deforestación del arroyo central desde su naciente y la ocupación de sus márgenes fueron los causantes de esta desforestación. Por ejemplo, alguno de sus trechos sufren con la ocupación inadecuada de edificios comerciales, con el lanzamiento de escombros y basuras etc. (Municipio de Sorriso, 2007).

Entre 1998 y 2003, el municipio registro 7.922 focos de calor (quemadas) uno de los valores más altos dentro del Estado de Mato Grosso. Los datos que aparecen en la tabla 5 muestran las principales consecuencias sociales vinculadas al desarrollo del municipio de Sorriso en el período de 1991 al 2000:

En el año de 2000 el Producto Interno Bruto (PIB) per cápita equivalía aproximadamente a $\mathrm{R} \$ 11.189$, superior al registrado en Brasil y al del Estado de Mato Grosso (R\$ 5.300 y R\$ 6.387) respectiva- 
mente. El Índice de Desarrollo Humano (IDH) es superior a la media del Estado y de Brasil, o sea, el IDH de Sorriso en el 2000 fue de 0,824 y este mismo IDH para el Estado de Mato Grosso y Brasil fue de 0,733 y 0,766 respectivamente; el aumento del ingreso medio per cápita del 1er quinto más pobre de la población en $\mathrm{R} \$ 15.35$, aproximadamente $31 \%$; el aumento de la desigualdad en la distribución de los ingresos reflejado en el aumento del índice de Gini. En Sorriso este índice creció $12,17 \%$, siendo este un valor muy superior al registrado en el Estado y en Brasil cuyos valores reflejan un acrecentamiento del 5,35\% y 1,74\% respectivamente; la disminución de $0,65 \%$ de los ingresos apropiados por el $20 \%$ más pobre; el aumento de $7,96 \%$ de los ingresos apropiados por el 20\% más rico; el aumento de 56,09\% de la relación existente entre el ingreso recibido por el $10 \%$ de los más rico y el $40 \%$ de los más pobre. Lo que representa una magnitud superior a la observada en el Estado y en Brasil en 2,8 y 6,8 respectivamente; el crecimiento de 42,61\% de la relación existente entre el ingreso recibido por el $20 \%$ de los más ricos y el $40 \%$ de los más pobre. O sea, una relación 2,58 veces superior a la registrada en el Estado y 6,22 veces superior a la registrada en Brasil.

Los resultados mostrados en los ítems “a”, “b” y “c” representan los argumentos utilizados por los defensores del agronegocio, para demostrar las ventajas sociales producidas por los grandes latifundios productores de soja y mostrar el proceso de expansión/ocupación del municipio como uno de los ejemplos positivos de la aplicación de las políticas públicas neoliberales dentro del proceso de globalización en la región.

Los resultados exhibidos en los ítems “d”, “e”, “f”, “g” y "h” demuestran que ese desempeño de la economía del municipio esta acoplado a un fuerte proceso de concentración y centralización de los ingresos el cual le garantiza grandes privilegios para una pequeña minoría que conforma la elite da producción de soja. Dicho de otra forma, en el año 2000, el primer quinto más pobre de la población del municipio recibía como ingresos per cápita medios $\mathrm{R} \$ 65$ y la décima parte más rica $\mathrm{R} \$ 2.550 .00$, o sea, la diferencia de ingresos entre ambos extremos es del $3.907,60 \%$. Por otro lado el porciento de los ingresos apropiados por el $20 \%$ de la población más pobre del municipio disminuyó en 0,65\% en el período de 1991 a 2000 y el porcentaje de los ingresos apropiado por el 10\% más rico creció en $10,64 \%$ en el municipio.

Estos indicadores muestran que las políticas públicas utilizadas para ocupar/expandir la actividad económica del municipio de Sorriso, apoyadas en la producción de soja (en grandes plantaciones) condujo a un fuerte proceso de concentración de la riqueza y consecuentemente a la exclusión de aquellas familias que no se integran y/o adaptan a este "modelo de desarrollo" 
Tabla 5

Resumen de indicadores sociales en período de 1991-2000 en Brasil, Mato Grosso y Sorriso

\begin{tabular}{|c|c|c|c|c|c|c|c|c|c|c|}
\hline \multirow[b]{2}{*}{$\mathbf{N}^{\circ}$. } & \multirow[b]{2}{*}{ INDICADORES } & \multicolumn{3}{|c|}{1991} & \multicolumn{3}{|c|}{2000} & \multicolumn{3}{|c|}{ Variación 1991/00 (\%) } \\
\hline & & MT & Sorriso & BR & MT & Sorriso & BR & MT & Sorriso & BR \\
\hline 1 & PIB per cápita (RS) & -- & -- & 0,08 & 5,300 & $11,189^{*}$ & 6,387 & -- & - & -- \\
\hline 2 & Indice de Gini & 0,598 & 0,567 & 0,634 & 0,630 & 0,636 & 0,645 & 5,35 & 12,17 & 1,74 \\
\hline 3 & $\begin{array}{l}\text { Ingreso per cápita medio del } 1^{\circ} \\
\text { quinto más pobre }\end{array}$ & 27,63 & 49,93 & 21,83 & 30,23 & 65,28 & 22,23 & 109,4 & 130,74 & 101,8 \\
\hline 4 & $\begin{array}{l}\text { Ingreso per cápita medio del décimo } \\
\text { más rico }\end{array}$ & 1007,49 & 1280,75 & 1174,21 & 1525,4 & 2550,94 & 1556,24 & 151,40 & 199,18 & 132,53 \\
\hline 5 & $\begin{array}{l}\% \text { del ingreso apropiado por el } 20 \% \\
\text { más pobre }\end{array}$ & 2,70 & 3,48 & 1,90 & 2,10 & 2,43 & 1,50 & $-0,60$ & $-0,65$ & $-0,40$ \\
\hline 6 & $\begin{array}{l}\% \text { del ingreso apropiada por el } 20 \% \\
\text { más rico }\end{array}$ & 64,12 & 60,47 & 67,11 & 67,03 & 68,43 & 68,06 & 2,91 & 7,96 & 0,95 \\
\hline 7 & $\begin{array}{l}\% \text { del ingreso apropiado por el } 10 \% \\
\text { más rico }\end{array}$ & 49,18 & 44,65 & 50,99 & 52,95 & 55,29 & 52,36 & 3,77 & 10,64 & 1,73 \\
\hline 8 & $\begin{array}{l}\text { Relación entre el ingreso del } 10 \% \\
\text { más rico y el } 40 \% \text { más pobre }\end{array}$ & 22,44 & 17,08 & 30,43 & 26,92 & 26,66 & 32,93 & 19,96 & 56,09 & 8,22 \\
\hline 9 & $\begin{array}{l}\text { Relación entre el ingreso del } 20 \% \\
\text { más ricos y el } 40 \% \text { más pobres }\end{array}$ & 14,63 & 11,57 & 20,03 & 17,04 & 16,50 & 21,40 & 16,47 & 42,61 & 6,84 \\
\hline 10 & $\begin{array}{l}\text { Indice de Desarrollo Humano } \\
\text { (Longevidad) }\end{array}$ & 0,654 & 0,712 & 0,662 & 0,740 & 0,805 & 0,727 & 13,15 & 13,06 & 9,82 \\
\hline 11 & $\begin{array}{l}\text { Indice de Desarrollo Humano } \\
\text { (Educación) }\end{array}$ & 0,741 & 0,797 & 0,745 & 0,860 & 0,869 & 0,849 & 16,06 & 9,03 & 13,96 \\
\hline 12 & $\begin{array}{l}\text { Indice de Desarrollo Humano } \\
\text { (Ingreso) }\end{array}$ & 0,661 & 0,717 & 0,681 & 0,718 & 0,797 & 0,723 & 8,62 & 11,16 & 6,17 \\
\hline 13 & $\begin{array}{l}\text { Indice de Desarrollo Humano (IDH y } \\
\text { IDH-M) }\end{array}$ & 0,685 & 0,742 & 0,696 & 0,733 & 0,824 & 0,766 & 12,74 & 11,10 & 10,11 \\
\hline
\end{tabular}

Fuente: Elaboración propia a partir del Atlas de Desenvolvimento Humano do Brasil.

Este simple ejercicio analítico nos conduce a repensar sobre la "sostenibilidad" de ese modelo de desarrollo dirigido por el factor económico pero que es negligente con la racionalidad ambiental y social. En este sentido compartimos la opinión de Schlesinger y Noronha (2006) cuando destacan la necesidad urgente de una agenda de políticas que beneficien otras actividades económicas menos intensivas en recursos naturales y con mayor valor agregado y menor concentración de los ingresos. Además es necesario y decisivo destacar que en una sociedad democrática deben existir mayores espacios de participación y de interacción para que las personas puedan elegir el tipo de desarrollo que ellos desean.

\section{Necesidad de busca y aplicación de "nuevas" políticas públicas de estímulo y "nuevos” modelos organizacionales: la importancia del enfoque de arreglos y sistemas productivos innovadores locales}

Con el proceso de apertura comercial y liberalización de la economía a partir de los años 90, se aceleran los mecanismos de busca de "políticas de promoción” y “modelos organizacionales” que generen condicio- 
nes de competitividad a los diferentes actores e instituciones en un "nuevo" ambiente, el cual exige: creciente capacidad propia de captación de recursos, agilidad y flexibilidad para responder a las demandas y fuerte capacidad de monitoreo de su entorno (científico, económico, social, etc.).

Así, la visión y el énfasis en el enfoque de arreglos y sistemas productivos innovadores locales puede y debe convertirse en una herramienta básica en el análisis del papel a desempeñar por las micros, pequeñas y medianas empresas (en el desarrollo del territorio) capaces de exhibir procesos de aprendizaje interactivo, cualificaciones técnicas y organizativas, conocimientos tácitos acumulados, fiducia en las relaciones y la proximidad geográfica, social y cultural como fuentes importantes de diversidad y ventajas comparativas, así como las formas de promoverlas. (Lemos, 2003).

Según Cassiolato (Cassiolato et. al 2003a; 2003b) este enfoque es importante por ser apropiado para promover y orientar políticas que estimulen el desarrollo de las aglomeraciones productivas locales, estimulando las relaciones de interacción, la promoción del aprendizaje y absorción de conocimiento entre los diferentes actores con proximidad geográfica. Es decir cada vez más crece la tendencia al cambio en las formas de formular las políticas públicas, así como la necesidad de apreciar las mismas bajo un prisma holístico, donde se reconoce la importancia: 1) de los procesos interactivos de innovación y aprendizaje y su carácter local, así como, 2) el enfoque de sistemas nacionales de innovación, lo que hace que estos procesos sean objeto de preocupaciones por parte de los formuladores de políticas en muchos países (Lemos, 2003).

De esta forma, las políticas de "promoción" de la innovación se tornan actualmente mucho más importantes que en el pasado, teniendo en vista el reconocimiento crucial para intensificar la competitividad, a través del fortalecimiento de la capacidad de los individuos y las empresas de aprender y adquirir conocimientos. Según Lemos (Ibid) es necesario pensar e instrumentalizar las nuevas políticas y estrategias de desarrollo adoptando el enfoque sistémico, conforme el concepto de sistemas nacionales de innovación (SNI) que desde una perspectiva amplia de análisis, puede ser vista como una herramienta de análisis y de promoción del desarrollo y del crecimiento económico.

La noción de SNI se constituye en una de las mejores estructuras para capturar y enfrentar los diferentes desafíos actuales de la "llamada" globalización financiera, fase asentada en el conocimiento y en el aprendizaje para la integración de las estrategias que combinan innovaciones técnicas, organizacionales e institucionales (Ibid). Además el uso de este concepto permite realizar los vínculos entre las condiciones macroeconómicas de un determinado país y las necesidades microeconómicas específicas de los arreglos locales particulares. Derivado de esto puede se establecer una mayor coherencia y articulación entre las políticas implícitas y explicitas, además de evitar que las primeras anulen por completo la implementación de las segundas (Lemos 2003). 
Los objetivos de las recomendaciones de política en este enfoque van dirigidas a la necesidad de que los formuladores de política pública tengan presente desde una amplia perspectiva el concepto de innovación y de las oportunidades de aprendizaje e innovación en las micro, pequeñas y medianas empresas que interactúan en actividades tradicionales y regiones menos desarrolladas. O sea, no debemos considerar la promoción de la innovación y del desarrollo regional y social como objetivos excluyentes.

Para la incorporación de esos objetivos de forma convergente en los diferentes municipios que conforman el Estado de Mato Grosso se hace necesario ampliar la noción de innovación no restringiéndola a algo que sea totalmente nuevo en el mundo, originada única y exclusivamente de los procesos de investigación y desarrollo (I+D) y dirigida para ambientes tecnológicamente más avanzados o de gran complejidad tecnológica, cualquier aglomerado, así como los diversos actores que en el actúan pueden ser locus de innovación y objetos de políticas, de iniciativas de promoción de la innovación y del desarrollo sean ellos más o menos articulados, rudimentarios o más complejos (Ibid).

La noción de sistemas de innovación significa una nueva perspectiva para ampliar el conjunto de políticas: sociales, de mercado de trabajo, educación, industriales, ambientales de C y T entre otros. Este concepto impone el desarrollo de nuevas estrategias nacionales asociadas a la coordinación precisa de todas las áreas de política que afectan el proceso de aprendizaje y la construcción de competencias. Por tanto uno de los elementos a tener presente es la construcción de competencias a largo plazo, incluyendo todas estas áreas y todos sus objetivos comunes.

Entendemos que resulta adecuada la adopción del concepto de sistemas de innovación y en particular su dimensión regional o local, para promover las innovaciones en los aglomerados productivos conformados por empresas de tamaño micro, pequeño y medio, lo que refuerza la importancia de la difusión del conocimiento y el aprendizaje interactivo en estos sistemas. O sea, a partir de este enfoque podríamos identificar las necesidades específicas de este APL (o aglomeración), sus insuficiencias en relación con la innovación, la proximidad regional y la interacción, para poder descubrir los aspectos tácitos latentes en los mismos.

En el caso especifico del APL de la soja del municipio de Sorriso, la investigación de campo verificó la presencia de escasos vínculos significativos de interacción, cooperación y aprendizaje de forma tal que este aglomerado de productores está lejos de ser considerado un sistema de producción e innovación local como el descrito en este ítem. Utilizando la tabla 6 como base de análisis describimos la participación y/o conocimiento de los mismos sobre los programas o actividades dirigidas para la promoción de los APL, o sea, cuando los empresarios de la 
soja fueron cuestionados en relación a esa promoción respondieron así:

En relación a los programas del Gobierno Federal: 62\% conocen y participan y 38\% conocen y no participan; en cuanto a programas del Gobierno Estatal: 33\% conocen y participan y 38\% conocen y no participan; en cuanto a programas y acciones del Gobierno municipal: 29\% conocen y participan, 19 conocen y no participan y 52\% no conocen; En programas de otras instituciones: $17 \%$ conocen y participan, $17 \%$ conocen y no participan y 66\% no conocen. Lo anterior significa que (exceptuando los programas del Gobierno Federal) en promedio el 75\% de los empresarios de la soja entrevistados no participa en programas y acciones de promoción de APL en el municipio de Sorriso.

\section{Tabla 6}

\section{Participación y/ o conocimiento sobre algún tipo de programa o acciones dirigidas para MPEs}

\begin{tabular}{lccc}
\hline \multicolumn{1}{c}{ Institución } & \multicolumn{3}{c}{ Microempresas } \\
\cline { 2 - 4 } & No conoce & Conoce, mas no & Conoce y \\
& & participa & participa \\
1. Gobierno Federal & $0,0 \%$ & $38,1 \%$ & $61,9 \%$ \\
2. Gobierno Estatal & $42,9 \%$ & $23,8 \%$ & $33,3 \%$ \\
3. Gobierno Local/Municipal & $52,4 \%$ & $19,0 \%$ & $28,6 \%$ \\
4. Otras Instituciones & $66,7 \%$ & $16,7 \%$ & $16,7 \%$ \\
\hline
\end{tabular}

Fuente: Trabajo de campo propio.

La tabla 7 muestra los criterios emitidos por los empresarios de la soja en relación a la evaluación de los programas o acciones especificas para el segmento en que ellos actúan, esto es: En relación a los programas del Gobierno Federal: 25\% lo evaluaron positivamente, 60\% proporcionaron nota negativa y el 15\% no tenían elemento para la evaluación; en Cuanto programas del Gobierno Estatal:19\% lo evaluó positivamente, 24\% dieron nota negativa y 57\% no lo evaluó; en cuanto a programas y acciones del Gobierno municipal: 29\% lo evaluó positivamente, 24\% negativamente y $48 \%$ no lo evaluó; respecto de programas de otras instituciones: $25 \%$ dio nota negativa y $75 \%$ no tenía criterios para evaluarlo. En promedio el 33\% de los empresarios que participan de programas y acciones dirigidas a la promoción de APLs evaluaron negativamente las mismas y 60\% se considero sin elementos para la evaluación. 
Tabla 7

Evaluación de los programas o acciones específicas para el segmento en que actúa las MPEs

\begin{tabular}{lccc}
\hline \multicolumn{1}{c}{ Institución } & \multicolumn{3}{c}{ Micro } \\
\cline { 2 - 4 } & Evaluación & Evaluación & Sin elementos \\
& Positiva & Negativa & para Evaluación \\
1. Gobierno Federal & $25,0 \%$ & $60,0 \%$ & $15,0 \%$ \\
2. Gobierno Estatal & $19,0 \%$ & $23,8 \%$ & $57,1 \%$ \\
3. Gobierno Local/Municipal & $28,6 \%$ & $23,8 \%$ & $47,6 \%$ \\
4. Otras Instituciones & $0,0 \%$ & $25,0 \%$ & $75,0 \%$ \\
\hline
\end{tabular}

Fuente: Pesquisas de campo propio

Por otra parte cuando cuestionados en relación con la importancia de las Políticas Publicas que contribuyen en el aumento de la eficiencia del APL destacaron por orden decreciente de importancia las siguientes (ver figura 5). a) Los programas de capacitación profesional y entrenamiento técnico: esto debe ser entendido como la necesidad de combinar el conocimiento tácito local existente y las formas de transferencia de los mismos con la capacitación de la mano de obra contratada; b) mejora en la educación básica; c) necesidad de líneas de crédito; d) programas de acceso a la información, que le permitan dominar mejor las técnicas de producción, marketing y comercialización de sus productos; e) programas de estímulos a las inversiones; f) necesidad de incentivos fiscales: todos se quejaron de la alta carga tributaria que debe ser paga desde la siembra hasta la entrega de la soja para la comercialización, lo que disminuye sus ingresos.

\section{Figura 5}

\section{Políticas públicas que contribuyen para el aumento da eficiencia competitiva das MPEs}

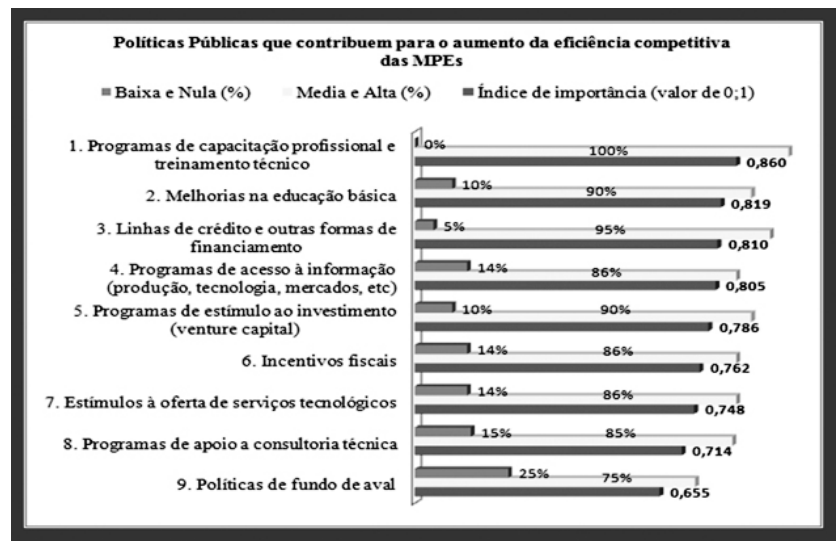


A modo de resumen debemos destacar la importancia que representa trabajar con esta noción de APL y SPIL dentro del municipio en el sentido que el desafío fundamental radica en saber lidiar con los diferentes agentes locales de forma colectiva y con una perspectiva sistémica. Siendo así, el éxito de estas políticas de promoción del desarrollo dependerá de cuanto mayor sea el foco en el conjunto de agentes.

Según Lemos (2003) las dificultades de programar y promover estos formatos serán cada vez mayores, porque es más complicado diseñar e implementar políticas dirigidas para un conjunto de actores que para empresas particularmente. Este ejercicio demanda esfuerzos adicionales e inéditos de operalización y requiere de una política con visión sistémica para construir nuevos referenciales que agrupen los varios aspectos correlacionados, incluyendo aquellos relacionados con la tributación, regulación y legislación dentro del municipio.

Debemos tener presente que establecer y desarrollar acciones en APLs y SPILs significa mucho más que dar acceso a los servicios tecnológicos, comerciales, financieros, jurídicos etc., o ampliar los recursos disponibles, para las empresas localizadas en el municipio. Implica un esfuerzo de rediseñar instrumentos y de reconocer la diversidad y la especificidad dentro del APL y/oSPILs. O sea, requiere, por parte de los organismos que pretenden formular e implementar acciones en APL y SPILs, un aprendizaje institucional dirigido para una nueva forma de pensar y de hacer política que permita lidiar con todos estos aspectos, los cuales no están presentes en el municipio objeto de investigación.

\section{Consideraciones finales}

Consideramos que las políticas de promoción deben enfatizar la importancia del desarrollo de los recursos humanos y la integración de las diferentes partes de los sistemas de innovación en redes así como la interacción. En el caso del municipio de Sorriso, el gran desafío es la medición y análisis de los procesos de polución y degradación ambiental. El desarrollo y/o adopción de tecnologías y practicas ecológicas que preserven los ecosistemas allí existentes y el estimulo a las innovaciones que alternen incentivos a la agricultura y a la silvicultura así como técnicas favorables al medio ambiente son indispensables. Innovaciones sociales que disminuyan la desigualdad en el modo de vida de la población en su día a día, constituyéndose en elementos valiosos en la estrategia de desarrollo sostenible. 


\section{Notas}

${ }^{1}$ Se entiende por Sistema Productivos Innovativos Locales (SPILs) al conjunto de agentes económicos, políticos y sociales localizados en un mismo territorio, desarrollando actividades económicas conjuntas los cuales presentan vínculos expresivos de producción, interacción, cooperación y aprendizaje. Los SPILs generalmente incluyen empresas productoras de bien y servicios finales, suministradoras de equipamientos y otros insumos, prestadoras de servicio, comercializadoras, clientes, etc., cooperativas asociaciones y representaciones y demás organizaciones dedicadas a la formación y entrenamiento de recursos humanos, información, investigación + desarrollo (I+D), promoción y financiamiento. Los Arreglos Productivos Locales (APLs) son aquellos casos que no presentan significativa articulación entre los diferentes agentes y que siendo así no pueden caracterizarse como sistemas.

${ }^{2}$ Silva, L.: O papel do Estado no processo de ocupação das áreas de Cerrado entre as décadas de 60 e 80. Em: Caminhos de geografia - Revista Online, dezembro de 2000, pp. 30-34.

${ }^{3}$ Ver www.agricultura.gov.br/sarc/dfpv/prodecer.htm

${ }^{4}$ Año-zafra o Año agrícola - período de tiempo existente entre la fase de siembra y la cosecha (especialmente de granos y cereales) y no necesariamente coincide con el período de 12 meses existentes entre enero y diciembre.

${ }^{5}$ Considerando o ICMS, na LEI COMPLEMENTAR No 87 (1996), Art. $3^{\circ}$ O imposto não incide sobre:

II - operações e prestações que destinem ao exterior mercadorias, inclusive produtos primários e produtos industrializados semi-elaborados, ou serviços;

Parágrafo único. Equipara-se às operações de que trata o inciso II a saída de mercadoria realizada com o fim específico de exportação para o exterior, destinada a:

I - empresa comercial exportadora, inclusive tradings ou outro estabelecimento da mesma empresa;

II - armazém alfandegado ou entreposto aduaneiro.

${ }^{6}$ Corte raso es un término técnico que, en silvicultura, significa cortar por la base todos los arboles de una determinada área.

${ }^{7}$ El fuego es usado en la abertura y el manejo de pastos en la agricultura el "corte y quema” generalmente se escapa al control, quemando culturas perenes, sistemas agro forestales e florestas manejadas para la producción maderera. Estas pérdidas estimulan a los productores a continuar optando por sistemas de producción extensivos, manteniendo su dependencia del fuego.

$8 \frac{38097}{5770+5463}=339$ hectares contra $\frac{23463}{16370}=143$ hectareas en promedio

${ }^{9}$ El Arroyo Central pertenece a la Cuenca Amazónica (Mapa Hidrográfico A11 SEMA-MT) y corta el centro de la ciudad. Su extensión es de aproximadamente 2,3 km con su naciente localizada próxima a la Praça Marco Zero, yendo a desaguar en el Rio Tenente Lira que, a su vez, desagua en el Rio Teles Pires. 


\section{Bibliografía}

Bickel, U. (2004), Brasil: Expansão da Soja, Conflitos Sócio-Ecológicos e Segurança Alimentar, Tesis de Maestria, Faculdade de Agronomia. Universidad de Bonn, Bonn.

Carvalho, H. (2004), A questão agrária e o fundamentalismo neoliberal no Brasil, Curitiba, 12 de maio de 2004. Disponible em: http://resistir.info/ brasil/questao_agraria.html

Cassiolato, J. y Lastres, H. (2003a), Novas Políticas na Era do Conhecimento: O foco em APLs, IE/UFRJ Disponible en: http:// www.ie.ufrj.br/redesist

Ídem (2003b), Políticas para a promoção de APLs. de micro e pequenas empresas: conceitos, vantagens e restrições do e equívocos usuais, IE/ UFRJ, disponible en: http://www.ie.ufrj.br/redesist

IBGE (2009), Sistema IBGE de Recuperação Automática (SIDRA), disponible en: http://www.sidra.ibge.gov.br/bda/tabela/protabl1.asp. Acesso 0703-2009

IPAM (2004), Desmatamento na Amazônia: indo além da emergência crônica, disponible en http://www.amazonia.org.br

IPEA (2000), Atlas de Desenvolvimento Humano do Brasil, disponible en: http://www.pnud.org.br

Jornal Folha de Sao Paulo (2006), Soja desmata a Amazônia, comprova dado de satélite, en Caderno Ciência. 05 de setembro de 2006.

Lemos, C (2003), Micro, Pequenas e Médias Empresas no Brasil: Novos requerimentos de Políticas para a promoção de Sistemas Produtivos Locais: Tesis de Doctorado UFRJ, Rio de Janeiro.

Mançano, B.(2004), Agronegócio e Reforma Agrária, Disponible em: http:/ /www.mst.org.br/mst/pagina.php?cd=501

Melo, J. (2009), Qual é o determinante da Expansão da Fronteira Agrícola Mato-grossense no período 2001/2007: Produção Agrícola Ou Pecuária?, ponencia presentada en la Faculdad de Economía, Universidad Federal de Mato Grosso, Cuiabá.

Méndez del Villar, P. (2005), Impacto do Projeto de Asfaltamento da BR163: Perspectivas da Sociedade e do Setor Produtivo Agrícola ao longo do eixo da Cuiabá - Santarém, documento inédito.

Reydon, P.B. y Cornelio F. (2006), Mercados de Terras no Brasil: Estrutura e Dinâmica, Ministerio de Desenvolimento Agrario, NEAD, Brasilia. 
Municipio de Sorriso (2007), "Nativa engenharia e meio ambiente, Diagnóstico Ambiental do Córrego Central do Município de Sorriso" en Relatorio Tecnico $n^{\circ}$ 016/2007, Municipio de Sorriso, Sorriso.

Nepstad, D. (2000), Avança Brasil: Os custos ambientais para a Amazônia. Relatório do Projeto: Cenários futuros para a Amazônia, Instituto de Pesquisa Ambiental da Amazônia (IPAM), Belém,

Schlesinger S. y Noronha, S (2006), O Brasil está nu!. O avanço da monocultura da soja o grão que cresceu demais, Ediciones Federação de Órgãos para Assistência Social e Educacional (FASE), Rio de Janeiro.

SEPLAN-MT (2009), Zoneamento Sócio Econômico Ecológico do Estado de Mato Grosso 2002/2003, disponible en: http:// www.zsee.seplan.mt.gov.br/servidordemapas/Run.asp . Acesso em 01/jun/ 2009

Recibido: 23.08.2011

Aceptado: 23.11.2011 